\title{
ON ILL-POSEDNESS OF FREE-BOUNDARY PROBLEMS FOR HIGHLY COMPRESSIBLE TWO-DIMENSIONAL ELASTIC BODIES
}

\author{
YU. V. EGOROV AND E. SANCHEZ-PALENCIA
}

To V. M. Babich on the occasion of his 80th birthday

\begin{abstract}
Some problems of elasticity theory related to highly compressible twodimensional elastic bodies are considered. Such problems arise in real elasticity and pertain to some materials having negative Poisson ratio. The common feature of such problems is the presence of a small parameter $\varepsilon$. If $\varepsilon>0$, the corresponding equations are elliptic and the boundary data obey the Shapiro-Lopatinsky condition. If $\varepsilon=0$, this condition is violated and the problem may fail to be solvable in distribution spaces. The rather difficult passing to the limit is studied.
\end{abstract}

\section{INTRODUCTION}

Elliptic boundary value problems are nowadays well studied and take a very important place in the modern theory of partial differential equations. Their theory is based on the so-called Shapiro-Lopatinsky conditions (SL) that give an algebraic reformulation of the normal solvability of boundary value problems. (See, for example, [1, Chapter 2, 2].)

Usually, violation of SL leads to a very difficult problem and demands new methods. Here we consider some examples of this kind, arising in real elasticity problems (see also [3]-5] and the references therein). These examples pertain to some materials having negative Poisson ratio (see [2, 6, 7]). Actually, they are merely models, but they show the specific character of the problems in question and the nature of the results in real situations.

Moreover, such situations arise in many other problems of mathematical physics. The principal feature of these problems is the necessity to consider elliptic boundary value problems with the Shapiro-Lopatinsky condition violated. This implies the unsolvability of the problems in distribution spaces. To solve such problems, it is necessary to consider generalized functions with exponentially growing Fourier transforms.

The common property of such problems is the fact that they involve a small parameter $\varepsilon$ and reduce to an equation of the form

$$
\left(A^{0}+\varepsilon A^{1}\right) w^{\varepsilon}=f
$$

with $A^{0}$ generically smoothing and $A^{1}$ elliptic. The main role in the asymptotic expansion of solutions as $\varepsilon$ tends to 0 is played by the Fourier components with frequencies that are $O\left(\log \left(\varepsilon^{-1}\right)\right)$, and the convergence to the limit is rather slow.

Similar phenomena were observed by Babich and Buldyrev in the book 8$]$.

2010 Mathematics Subject Classification. Primary 35R25, Secondary 74B99.

Key words and phrases. Two-dimensional elasticity, negative Poisson ratio, elliptic boundary value problems.

(C)2011 American Mathematical Society 


\section{§1. TWO-DIMENSIONAL ELASTICITY \\ WITH SMALL RIGIDITY FOR COMPRESSION}

We shall consider two-dimensional elastic media with small rigidity against uniform compression. The limit case with zero rigidity corresponds to the situation where the Shapiro-Lopatinsky condition is not satisfied and the coercivity is lost.

Here, the small parameter of perturbation is the rigidity for the uniform compression. The perturbation term has the same order as the other terms, so the perturbation is not singular. This problem is similar to that considered in [3], but the singular part of the perturbation is of smaller order than therein.

Since we consider two-dimensional media, the indices $\alpha, \beta, \gamma, \ldots$ take the values $\{1,2\}$. Let

$$
u=\left(u_{1}, u_{2}\right)
$$

be the displacement vector, let

$$
e_{\alpha \beta}=\frac{1}{2}\left(\partial_{\alpha} u_{\beta}+\partial_{\beta} u_{\alpha}\right)
$$

be the strain tensor, and let the deviation tensor have the components

$$
\tilde{e}_{\alpha \beta}=e_{\alpha \beta}-\frac{1}{2} e_{\lambda, \lambda} \delta_{\alpha \beta} .
$$

The bilinear form of the energy is

$$
a(u, v)=\int_{\Omega}\left(2 \mu \widetilde{e}_{\alpha \beta}(u) \widetilde{e}_{\alpha \beta}(v)+K \operatorname{div} u \cdot \operatorname{div} v\right) d x,
$$

where $\mu>0$ and $K>0$ are the thermodynamic constants in the usual elasticity.

The limit case corresponds to the values $\mu>0$ and $K=0$, which means zero rigidity against uniform compression.

Using the stress tensor

$$
\sigma_{\alpha \beta}=K \operatorname{div} u \delta_{\alpha \beta}+2 \mu \tilde{e}_{\alpha \beta}(u),
$$

we can write the energy form as

$$
a(u, v)=\int_{\Omega} \sigma_{\alpha \beta}(u) e_{\alpha \beta}(v) d x .
$$

It is useful to write this in terms of the Poisson coefficients $\nu$ (we use Landau-Lifschitz's notation [2]). For this, we can write

$$
\tilde{e}_{\alpha \beta}=\frac{1}{4 K} \sigma_{\lambda \lambda} \delta_{\alpha \beta}+\frac{1}{2 \mu} \tilde{\sigma}_{\alpha \beta},
$$

where

$$
\tilde{\sigma}_{\alpha \beta}=\sigma_{\alpha \beta}-\frac{1}{2} \sigma_{\lambda \lambda}
$$

is the deviation. If $\sigma_{11}=1$ and $\sigma_{22}=\sigma_{12}=\sigma_{21}=0$, then

$$
\begin{aligned}
& e_{11}=\frac{1}{4 K}+\frac{1}{2 \mu}\left(1-\frac{1}{2}\right)=\frac{1}{4} \frac{K+\mu}{K \mu}, \\
& e_{22}=\frac{1}{4 K}-\frac{1}{2 \mu}\left(1-\frac{1}{2}\right)=\frac{1}{4} \frac{\mu-K}{K \mu},
\end{aligned}
$$

so that

$$
\nu=-\frac{e_{22}}{e_{11}}=\frac{K-\mu}{K+\mu} .
$$


In the general case of $\nu>0$, this explains why we define $\nu$ with the minus sign. But the case of $\nu<1$ is possible; the Poisson coefficient may take any values between 1 (for $\mu / K=0)$ and $-1($ for $\mu / K=\infty)$.

\section{§2. Study of the Limit CASE: $K=0$}

If $K=0$ and $\mu>0$, the energy form (1.4) depends only on the deviator $\widetilde{e}$ (and not on the entire $e$ ). This explains why the coercivity may be lost.

If $K=0$, then

$$
a(u, v)=\int_{\Omega}\left(e_{\alpha \beta}(u)-\frac{1}{2} \operatorname{div} u \delta_{\alpha \beta}\right)\left(e_{\alpha \beta}(v)-\frac{1}{2} \operatorname{div} v \delta_{\alpha \beta}\right) d x
$$

and

$$
\sigma_{\alpha \beta}=2 \mu\left(e_{\alpha \beta}(u)-\frac{1}{2} \operatorname{div} u \delta_{\alpha \beta}\right)
$$

Explicitly,

$$
\begin{aligned}
& \sigma_{11}(u)=2 \mu\left(1-\frac{1}{2}\right) \partial_{1} u_{1}-\mu \frac{1}{2} \partial_{2} u_{2}=\mu\left(\partial_{1} u_{1}-\partial_{2} u_{2}\right), \\
& \sigma_{12}(u)=\mu\left(\partial_{2} u_{1}-\partial_{1} u_{2}\right), \quad \sigma_{22}(u)=\mu\left(\partial_{2} u_{2}-\partial_{1} u_{1}\right) .
\end{aligned}
$$

We can see that

$$
\sigma_{11}=-\sigma_{22},
$$

which is natural: since the rigidity under uniform compression is zero, the corresponding stress is zero.

Let us study the elasticity system for $K=0$ :

$$
-\partial_{\alpha} \sigma_{\alpha \beta}(u)=f_{\beta} .
$$

This means that

$$
\begin{aligned}
& -\left(\partial_{1}^{2}+\partial_{2}^{2}\right) u_{1}=f_{1}, \\
& -\left(\partial_{1}^{2}+\partial_{2}^{2}\right) u_{2}=f_{2} .
\end{aligned}
$$

This system is obviously elliptic. Evidently, the Dirichlet boundary data $u_{1}=\psi_{1}$, $u_{2}=\psi_{2}$ obey the Shapiro-Lopatinsky condition. However, for the Neumann boundary conditions this is not the case.

Proposition 2.1. The Neumann boundary conditions do not satisfy the SL.

Proof. Indeed, after changing the variables in such a way that the outer normal has the direction $n=(1,0)$, as a solution we can take

$$
u_{\alpha}=U_{\alpha} e^{i \lambda y} e^{|\lambda| x} .
$$

The Neumann boundary conditions

$$
\sigma_{\alpha \beta} n_{\alpha}=0
$$

take the form

$$
\sigma_{11}(u)=0, \quad \sigma_{12}(u)=0,
$$

or, by (2.4),

$$
\partial_{1} u_{1}-\partial_{2} u_{2}=0, \quad \partial_{2} u_{1}+\partial_{1} u_{2}=0 .
$$

By (2.9), this takes the form

$$
|\lambda| U_{1}-i \lambda U_{2}=0, \quad i \lambda U_{1}+|\lambda| U_{2}=0 .
$$


The determinant of this system vanishes, the equations are proportional, and we can take $U_{2}=U_{1}\left(-i \frac{|\lambda|}{\lambda}\right)$ to get a nonzero solution, as required.

Observe that if $K=0$, then

$$
\begin{aligned}
a(u, v) & =\mu \int_{\Omega}\left(\sigma_{11}(u) e_{11}(v)+2 \sigma_{12}(u) e_{12}(v)+\sigma_{22}(u) e_{22}(v)\right) d x \\
& =\mu \int_{\Omega}\left[\sigma_{11}(u)\left(e_{11}(v)-e_{22}(v)\right)+2 \sigma_{12}(u) e_{12}(v)\right] d x \\
& =\mu \int_{\Omega}\left[\left(e_{11}(u)-e_{22}(u)\right)\left(e_{11}(v)-e_{22}(v)\right)+4 e_{12}(u) e_{12}(v)\right] d x
\end{aligned}
$$

Set

$$
l_{1}(u)=e_{11}(u)-e_{22}(u), \quad l_{2}(u)=2 e_{12}(u)
$$

Then

$$
a(u, v)=\mu \int_{\Omega}\left[l_{1}(u) l_{1}(v)+l_{2}(u) l_{2}(v)\right] d x
$$

and we have precisely the same structure as in [4] (where $l=\left(l_{1}, l_{2}\right)$ is a numerical vector) or in the shell problem (where $l$ is a triplet).

\section{$\S 3$. ON MATERIALS OF THE TYPE IN QUESTION}

Materials with $\nu=-1$ can be constructed with a suitable micro-structure; see Almgren [6] or Lakes [7.

The basic idea can be seen in the following micro-structure:
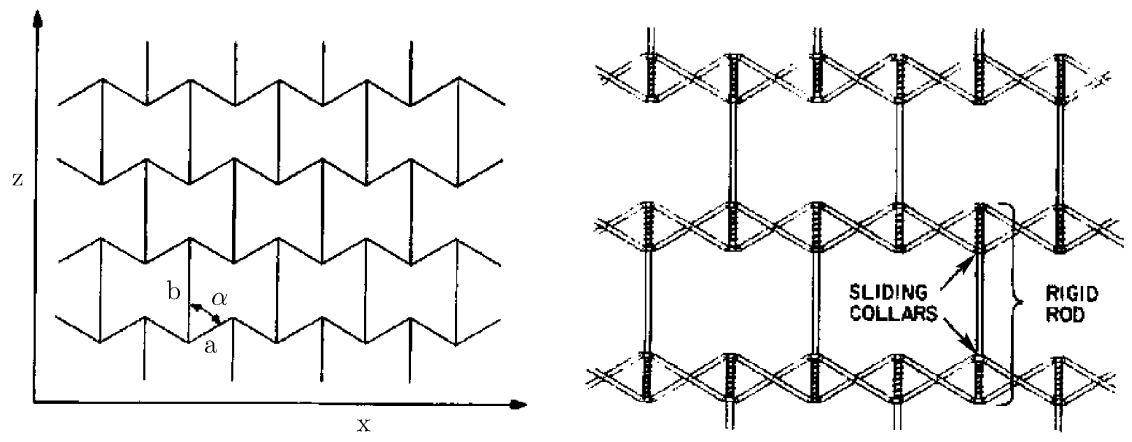

FiguRE 1. The micro-structure of the media

This construction is formed by rigid bars, the angle $\alpha$ is variable, so that $\alpha=\frac{\pi}{4}+$ $\delta \alpha$ and $\delta \alpha$, which can be positive or negative, is controlled with a spring. It is seen immediately that the horizontal and the vertical dilatations are equal, i.e., $\nu=-1$ or $\partial_{1} u_{1}=\partial_{2} u_{2}$.

It is possible to create different variants of this theme; for example, the rigid bars can be replaced with elastic ones. Structures of this type are often used for modeling foams.

Such structures are often anisotropic. We consider the anisotropic case in the next section.

The case where $K>0$ can be viewed as a small perturbation of the case where $K=0$. It is similar to the case of the boundary problem for $\Delta^{2}$ considered in [4, 5].

It is possible to study perturbations with a nonempty set of solutions of the equations

$$
l_{1}(u)=0, \quad l_{2}(u)=0,
$$


i.e.,

$$
\partial_{1} u_{1}-\partial_{2} u_{2}=0, \quad \partial_{2} u_{1}+\partial_{1} u_{2}=0 .
$$

\section{§4. Standard (anisotropic tWo-Dimensional) FrameWork}

4.1. As above, let

$$
u=\left(u_{1}, u_{2}\right)
$$

be the displacement vector, and

$$
e_{\alpha \beta}=\frac{1}{2}\left(\partial_{\alpha} u_{\beta}+\partial_{\beta} u_{\alpha}\right)
$$

the strain tensor. We have

$$
e_{11}=\partial_{1} u_{1}, \quad e_{12}=e_{21}=\frac{1}{2}\left(\partial_{2} u_{1}+\partial_{1} u_{2}\right), \quad e_{22}=\partial_{2} u_{2} .
$$

Let $a_{\alpha \beta \varphi \psi}$ be the elasticity coefficients, either real constants or functions of $x=\left(x_{1}, x_{2}\right)$. They have the following symmetry properties:

$$
a_{\alpha \beta \gamma \delta}=a_{\beta \alpha \gamma \delta}=a_{\alpha \beta \delta \gamma}=a_{\gamma \delta \alpha \beta} ;
$$

i.e., they are symmetric in $\alpha, \beta$ and in $\gamma, \delta$ and in the blocks $(\alpha \beta)$ with $(\gamma \delta)$. Note that it suffices to prescribe symmetry in $\alpha \beta$ and by blocks. Then the stress tensor is defined by

$$
\sigma_{\alpha \beta}=a_{\alpha \beta \gamma \delta} e_{\gamma \delta} .
$$

Obviously, $e$ and $\sigma$ are symmetric, and

$$
\sigma_{\alpha \beta}(u) e_{\alpha \beta}(v)=a_{\alpha \beta \gamma \delta} e_{\gamma \delta}(u) e_{\alpha \beta}(v)=e_{\alpha \beta}(u) \sigma_{\alpha \beta}(v) .
$$

Moreover, the elasticity coefficients must satisfy the positivity condition:

$$
a_{\alpha \beta \gamma \delta} \varphi_{\gamma \delta} \varphi_{\alpha \beta} \geq c\|\varphi\|^{2}
$$

with $c>0$ for any symmetric $\varphi$. If the coefficients are functions of $x$, then relation (4.6) is assumed to hold uniformly (i.e., $c=$ const).

Remark 4.1. The above hypotheses on the coefficients are preserved by change of orthonormal coordinates. Nonorthonormal coordinates are not used in the sequel. Covariant and contravariant components are the same, and indices may be located either above or below.

The system of equations of elasticity reads

$$
-\partial_{\alpha} \sigma_{\alpha \beta}(u)=f_{\beta} .
$$

It contains two equations and two unknowns $u_{1}, u_{2}$, each equation is of order 2 , and the total order is 4 .

Lemma 4.1. Under the above assumptons, system (4.7) is elliptic in the Petrovskiu sense.

Proof. Consider (4.7) with $f=0$ and constant coefficients (since ellipticity is a point property):

$$
0=-\partial_{\alpha} \sigma_{\alpha \beta}(u) \equiv-\partial_{\alpha} a_{\alpha \beta \gamma \delta} e_{\gamma \delta}(u) .
$$

We consider solutions of the form

$$
u=U e^{i\left(\xi_{1} x_{1}+\xi_{2} x_{2}\right)}
$$

with $\xi \in \mathbb{R}^{2} \backslash(0,0)$ and $U \in \mathbb{C}^{2}$. Ellipticity amounts to saying that (4.9) is a solution of (4.8) only if $U=(0,0)$. Solutions are periodic with periods $1 /\left|\xi_{1}\right|, 1 /\left|\xi_{2}\right|$. (The case where one of the $\xi_{j}$ vanishes will be considered later.) 
Multiplying (4.8) by $\bar{u}_{\beta}$ and integrating by parts over the period gives

$$
\begin{aligned}
0 & =-\int_{\mathrm{Per}} \partial_{\alpha} \sigma_{\alpha \beta}(u) \bar{u}_{\beta} d x=-\int_{\partial \mathrm{Per}} n_{\alpha} \sigma_{\alpha \beta}(u) \bar{u}_{\beta} d x+\int_{\mathrm{Per}} \sigma_{\alpha \beta}(u) \partial_{\alpha} \bar{u}_{\beta} d x \\
& =\int_{\mathrm{Per}} \sigma_{\alpha \beta}(u) e_{\alpha \beta}(\bar{u}) d x=\int_{\mathrm{Per}} a_{\alpha \beta \gamma \delta} e_{\gamma \delta}(u) e_{\alpha \beta}(\bar{u}) d x \geq c \int_{\mathrm{Per}}|e(u)|^{2} d x
\end{aligned}
$$

Then $e_{\alpha \beta}(u)=0 \Rightarrow \partial_{1} u_{1}=0 \Rightarrow U_{1}=0$, and similarly, $U_{2}=0$.

In the case where one of the $\xi_{j}$ vanishes, we consider periodicity only in the other variable, and integration over the corresponding period gives $u=U e^{i \xi_{2} x_{2}}$ (if $\xi_{1}=0$ ) and

$$
\partial_{2} u_{2}=0 \Rightarrow U_{2}=0, \quad \partial_{2} u_{1}+\partial_{1} u_{2}=0 \Rightarrow \partial_{2} U_{1}=0 \Rightarrow U_{1}=0 .
$$

In the sequel we consider the two kinds of boundary conditions: Dirichlet

$$
U_{1}=U_{2}=0
$$

or Neumann

$$
\sigma_{\alpha \beta} n_{\alpha}=0
$$

where $n$ is the outward unit normal.

Lemma 4.2. Under the hypotheses of the standard anisotropic framework, both kinds (Dirichlet or Neumann) of boundary data do satisfy the Shapiro-Lopatinsky condition.

Proof. The number of equations is 2 (and the total order of the system is 4 ). It is known (see, e.g., [5, S. 3]) that condition SL amounts to a well-posed (in the sense of unique existence) boundary value problem with constant (frozen) coefficients. The equation is given by the higher order terms, and the solution defined in the half-plane $x_{1}, x_{2}\left(x_{1}\right.$ is the tangential variable, $x_{2}$ is the inward normal), is equal to $e^{i \xi x_{1}}$, where $\xi \in \mathbb{R} \backslash\{0\}$ at $x_{2}=0$, and is decaying exponentially as $x_{2} \rightarrow \infty$. The problem is understood in the sense of "satisfying the equations in the half-plane and the boundary conditions at $x_{2}=0$ ". More precisely, since the number of conditions is 2 , the SL condition amounts to uniqueness with zero data.

Let $u=U\left(x_{2}\right) e^{i \xi x_{1}}$ be such a solution. It is periodic in $x_{1}$ of period $2 \pi /|\xi|$ and satisfies the system $0=-\partial_{\alpha} \sigma_{\alpha \beta}(u)$ (only the highest order terms are present). We multiply the equation by $\bar{u}_{\beta}$ and integrate over the "period" $P=(0,2 \pi /|\xi|) \times(0,+\infty)$, obtaining

$$
\begin{aligned}
0 & =-\int_{P} \partial_{\alpha} \sigma_{\alpha \beta}(u) \bar{u}_{\beta} d x=-\int_{\partial P} n_{\alpha} \sigma_{\alpha \beta}(u) \bar{u}_{\beta} d x+\int_{P} \sigma_{\alpha \beta}(u) \partial_{\alpha} \bar{u}_{\beta} d x \\
& =\int_{P} \sigma_{\alpha \beta}(u) e_{\alpha \beta}(\bar{u}) d x
\end{aligned}
$$

where the boundary integral vanishes: on the sides $x_{1}=0, x_{1}=2 \pi /|\xi|$ this happens by periodicity, on $x_{2}=0$ in the Dirichlet case this is because $u_{\beta}=0$ and in the Neumann case because $n_{\alpha} \sigma_{\alpha \beta}(u)=0$. (At infinity the integral vanishes exponentially.) We have also used symmetry. Finally, we have

$$
0=\int_{P} a_{\alpha \beta \gamma \delta} e_{\gamma \delta}(u) e_{\alpha \beta}(\bar{u}) d x,
$$

and since the coefficients (4.6) are positive, we see that $e_{\alpha \beta}(u)=0$ everywhere. It follows immediately that $u$ is a rigid displacement, i.e., a displacement generated by two translations along the axes and a rotation. But $u$ is of the form (4.9) so that $u=0$. 
4.2. Infinitely compressible elastic (two-dimensional) media. Now we consider a class of materials that do not fulfill the standard hypotheses.

We always consider real elastic coefficients $a_{\alpha \beta \gamma \delta}$ satisfying the symmetry conditions (4.4), but the positivity condition (4.6) is replaced by

$$
a_{\alpha \beta \gamma \delta} \varphi_{\gamma \delta} \varphi_{\alpha \beta} \geq 0
$$

for any symmetric $\varphi_{\alpha \beta}$. The above expression vanishes if and only if $\varphi$ is spherical, i.e.,

$$
a_{\alpha \beta \gamma \delta} \varphi_{\gamma \delta} \varphi_{\alpha \beta}=0 \Leftrightarrow \varphi_{\alpha \beta}=c \delta_{\alpha \beta},
$$

where $\delta_{\alpha \beta}$ is the Kronecker symbol, $c \in \mathbb{R}$.

In that case the corresponding $\sigma_{\alpha \beta}$ vanishes, i.e.,

$$
0=\sigma_{\alpha \beta}=a_{\alpha \beta \gamma \delta} \delta_{\gamma \delta}=a_{\alpha \beta \gamma \gamma}
$$

for any $\alpha, \beta$.

Remark 4.1. It is easily seen that the isotropic infinitely compressible medium considered above is a particular case of this one.

Otherwise, we emphasize that the symmetry hypotheses are preserved. In particular, symmetry by blocks $\left(a_{\alpha \beta \gamma \delta}=a_{\gamma \delta \alpha \beta}\right)$ is associated with the existence of an energy $\left(\frac{1}{2} a_{\alpha \beta \gamma \delta} e_{\gamma \delta} e_{\alpha \beta}\right)$, which is independent of the "history path" leading to the present state. In the present case, the energy vanishes precisely for $\varphi_{\alpha \beta}=c \delta_{\alpha \beta}$, since the corresponding $\sigma$ vanishes. Pure compressions (or expansions) are done with vanishing stress. Otherwise, the energy is positive.

Theorem 4.1. Under the hypotheses of the infinitely compressible two-dimensional materials, system (4.7) is elliptic in the sense of Petrovskiu.

Proof. The proof is the same as that of Proposition 2.1. At the end, using the (nonstrict) positivity of the coefficients (4.6), we only obtain

$$
e_{\alpha \beta}=c \delta_{\alpha \beta}
$$

for some $c \in \mathbb{C}$. This gives

$$
e_{12}(u)=0, \quad e_{11}(u)=e_{22}(u) .
$$

Our $u$ is of the form (4.9), so that

$$
i \xi_{2} U_{1}+i \xi_{1} U_{2}=0, \quad \xi_{1} U_{1}-\xi_{2} U_{2}=0,
$$

whence

$$
U_{1}=U_{2}=0
$$

Remark 4.2. In the present model, energy "cannot see" pure compressions (or dilations), but pure compressions are not involved in the ellipticity of the system, as we have shown. The proof is easy, but the result is not absolutely evident. This is the reason why we called it "Theorem 4.1", whereas the similar (well-known) property in the standard case was called "Proposition 2.1".

Concerning boundary conditions, we have the following statement.

Theorem 4.2. Under the hypotheses of the infinitely compressible two-dimensional materials,

i) the Dirichlet boundary data (4.10) satisfy the SL condition;

ii) the Neumann boundary data (4.11) do not satisfy the SL condition. 
Proof. The proof of i) is similar to that in the standard case. From (4.9) and (4.12) it follows that $e(u)$ is everywhere spheric, i.e.,

$$
\left\{e_{12}(u)=0, e_{11}(u)=e_{22}(u)\right\} \Leftrightarrow\left\{0=U_{1}^{\prime}\left(x_{2}\right)+i \xi U_{2}\left(x_{2}\right), i \xi U_{1}\left(x_{2}\right)=U_{2}^{\prime}\left(x_{2}\right)\right\},
$$

which gives

$$
U_{1}=C i \frac{\xi}{|\xi|} e^{-|\xi| x_{2}}, \quad U_{2}=C e^{-|\xi| x_{2}}
$$

for some complex constant $C$.

Furthermore, $u$ must satisfy the Dirichlet boundary condition (4.10) at $x_{2}=0$, yielding that $C=0$.

Now we prove ii).

We consider $u$ of the form (2.9). Obviously, it satisfies (2.10), so that, by (4.8), the corresponding $\sigma_{\alpha \beta}$ vanish everywhere. Therefore, they satisfy the equation and the (Neumann) boundary conditions at $x_{2}=0$; thus the problem in the half-space has a nonzero solution for zero data. The Shapiro-Lopatinsky condition is violated. Theorem 4.2 is proved.

4.3. Well-posed and ill-posed problems in the above frameworks. Elliptic problems with boundary data satisfying the Shapiro-Lopatinsky condition everywhere on the boundary are "well-posed" in the Agmon-Douglis-Nirenberg sense. This implies "existence and uniqueness" with two standard properties of regularity and continuity with respect to the data. Obviously, "existence" and "uniqueness" should be understood in certain cases "up to a finite-dimensional kernel" and "under compatibility conditions" for the data (a finite number of linearly independent conditions), respectively.

For instance, in the standard two-dimensional elasticity, for simply connected $\Omega$, with Dirichlet boundary conditions we have strict existence and uniqueness. For the Neumann problem, existence should be understood up to a three-dimensional kernel formed by the plane rigid displacements (two translations and one rotation), and existence only occurs under 3 linearly independent compatibility conditions. Applied forces must have vanishing resultant (2 conditions) and vanishing resultant moment (1 condition).

In the infinitely compressible case, it is obvious that, since the displacements generated by pure dilations or compressions (in the microscopic sense, i.e., with $e_{\alpha \beta}=c(x) \delta_{\alpha \beta}$ ) are "transparent" for stress, an elastic body "only may be held by the boundary conditions". The Dirichlet ones are "good", furnishing existence and uniqueness. The Neumann conditions are not suited, and the physical interpretation is clear.

A very interesting case appears when the boundary $\partial \Omega$ is made of two separated pieces $\partial \Omega=\Gamma_{0} \cup \Gamma_{1}$ and we prescribe

$$
\begin{array}{rll}
u_{\alpha} & =0 & \text { (Dirichlet) on } \Gamma_{0}, \\
\sigma_{\alpha \beta}(u) n_{\beta} & =0 & \text { (Neumann) on } \Gamma_{1} .
\end{array}
$$

Classically, this problem is well posed in the standard sense.

Hereafter, we shall show that in the infinitely compressible case the problem enjoys uniqueness, but, in general, not existence of the solutions, so it is ill-posed.

Indeed, the following is true.

Proposition 4.1. Under the hypotheses of two-dimensional infinitely compressible elastic media, the coefficients $a_{\alpha \beta \gamma \delta}$ are such that for any e,

$$
\sigma_{11}+\sigma_{22}=0
$$

or equivalently,

$$
a_{11 \alpha \beta}+a_{22 \alpha \beta}=0
$$


Proof. The tensor $\sigma=a e$ may be interpreted as a linear operator in $\mathbb{R}^{3} ; a$ is a symmetric matrix of size $3 \times 3$. The kernel is formed by $e=c I$. Then the range is orthogonal to the kernel, so that any $\sigma=a e$ is orthogonal to $e=c I$, i.e.,

$$
0=\sigma_{\alpha \beta} \delta_{\alpha \beta}=\sigma_{\alpha \alpha}=\sigma_{11}+\sigma_{22} .
$$

It follows that in the infinitely compressible case the system of equations (4.7), i.e.,

$$
-\partial_{1} \sigma_{11}(u)-\partial_{2} \sigma_{12}(u)=f_{1}, \quad-\partial_{1} \sigma_{12}(u)-\partial_{2} \sigma_{22}(u)=f_{2},
$$

takes the form

$$
-\partial_{1} \sigma_{11}(u)-\partial_{2} \sigma_{12}(u)=f_{1}, \quad-\partial_{1} \sigma_{12}(u)+\partial_{2} \sigma_{11}(u)=f_{2} .
$$

This system with the unknowns $u_{1}, u_{2}$ is formed by two equations, each of order 2 , and has total order 4. By Theorem 4.1, it is elliptic. Obviously, introducing the auxiliary unknowns $\sigma_{11}$ and $\sigma_{12}$ (see (4.5)), we see that (4.20) is equivalent to the system of 4 first order equations

$$
\begin{aligned}
-\partial_{1} \sigma_{11}-\partial_{2} \sigma_{12} & =f_{1}, \quad-\partial_{1} \sigma_{12}+\partial_{2} \sigma_{11}=f_{2}, \\
a_{11 \alpha \beta} e_{\alpha \beta}(u)-\sigma_{11} & =0, \quad a_{12 \alpha \beta} e_{\alpha \beta}(u)-\sigma_{12}=0,
\end{aligned}
$$

with the boundary conditions (4.14) and (4.15). Note that, since $\sigma_{22}=-\sigma_{11}$ was eliminated, (4.15) takes the form

$$
\sigma_{11} n_{1}+\sigma_{12} n_{2}=0, \quad \sigma_{12} n_{1}-\sigma_{11} n_{2}=0,
$$

which is equivalent to

$$
\sigma_{11}=0, \quad \sigma_{12}=0 .
$$

Then system (4.21), (4.22) with (4.14) or (4.15) can be viewed as two boundary value problems:

$$
\begin{aligned}
\operatorname{system}(4.21) \text { with } \sigma_{11} & =0, \quad \sigma_{12}=0 \text { on } \Gamma_{1}, \\
\text { system (4.22) with } u_{1}=0, & u_{2}=0 \text { on } \Gamma_{0} .
\end{aligned}
$$

The two "half-systems" form a "chain": the first is autonomous (two equations with two unknowns $\left.\sigma_{11}, \sigma_{12}\right)$ and the second consists of two equations for $u_{1}, u_{2}(\sigma$ are assumed to be known from the first "half-system").

Theorem 4.3. System (4.24) is an elliptic system of two equations for $\sigma_{11}$ and $\sigma_{12}$ of order 1 (total order 2) with Cauchy conditions on $\Gamma_{1}$.

System (4.25), where $\sigma_{11}$ and $\sigma_{12}$ are assumed to be known, is also an elliptic system of two equations for $u_{1}$ and $u_{2}$ of order 1 (total order 2) with Cauchy conditions on $\Gamma_{0}$.

Proof. The only nonobvious assertion is the ellipticity of the second system (4.24), i.e., the ellipticity in $u_{1}, u_{2}$ of the system

$$
a_{11 \alpha \beta} e_{\alpha \beta}(u)=\sigma_{11}, \quad a_{12 \alpha \beta} e_{\alpha \beta}(u)=\sigma_{12} .
$$

It is very easy to give an indirect proof of this property, because this system is "a part" of the elliptic system (4.7) (Lemma 4.1). Indeed, the ellipticity of (4.24) amounts to the existence of solutions for the system

$$
a_{11 \alpha \beta} e_{\alpha \beta}(u)=0, \quad a_{12 \alpha \beta} e_{\alpha \beta}(u)=0
$$

of the form $u=U e^{i\left(\xi_{1} x_{1}+\xi_{2} x_{2}\right)}$ with $\xi \in \mathbb{R}^{2} \backslash\{(0,0)\}$. Suppose that such a nonvanishing $u$ exists. By (4.26), the corresponding $\sigma_{11}$ and $\sigma_{12}$ should be zero, and $\sigma_{22}=-\sigma_{11}$. Then $u$ should be a solution of the equation $-\partial_{\alpha} \sigma_{\alpha \beta}(u)=0$, and this is impossible because this system is elliptic (Lemma 4.1). 
Thus, in the infinitely compressible case the elasticity system (4.7) with the boundary conditions (4.24), (4.25) amounts to successive solutions of two Cauchy elliptic problems.

\section{§5. Highly COMPRESSiBle TWO-DIMENSIONAL BODIES}

5.1. Generalities. As before, let the bilinear form of energy be

$$
a(u, v)=\int_{\Omega}\left[2 \widetilde{e}_{\alpha \beta}(u) \widetilde{e}_{\alpha \beta}(v)+\varepsilon \operatorname{div} u \cdot \operatorname{div} v\right] d x,
$$

where the shear modulus was taken equal to 1 , and $\varepsilon \geq 0$ denotes the modulus of rigidity to compression.

The stress tensor has the form

$$
\sigma_{\alpha \beta}=2 \widetilde{e}_{\alpha \beta}(u)+\varepsilon \operatorname{div} u \delta_{\alpha \beta},
$$

so that

$$
\begin{aligned}
& \sigma_{11}(u)=(1+\varepsilon) \partial_{1} u_{1}+(\varepsilon-1) \partial_{2} u_{2}, \\
& \sigma_{12}(u)=\partial_{2} u_{1}+\partial_{1} u_{2}, \\
& \sigma_{22}(u)=(\varepsilon-1) \partial_{1} u_{1}+(1+\varepsilon) \partial_{2} u_{2},
\end{aligned}
$$

and if $\varepsilon=0$, we have $\sigma_{11}+\sigma_{22}=0$. We can write the energy form as

$$
a(u, v)=\int_{\Omega} \sigma_{\alpha \beta}(u) e_{\alpha \beta}(v) d x .
$$

Consider a (two-dimensional) elastic body $\Omega$ with boundary $\partial \Omega=\Gamma_{0} \cup \Gamma_{1}$, where $\Gamma_{0}$ and $\Gamma_{1}$ are disjoint and smooth curves. We assume that the body is fixed on $\Gamma_{0}$, whereas $f=\left(f_{1}, f_{2}\right)$ is a field of forces applied to the free boundary $\Gamma_{1}$. The classical variational formulation of the elasticity problem is as follows:

$$
\text { to find } u^{\varepsilon} \in V \text { such that } a^{\varepsilon}\left(u^{\varepsilon}, v\right)=\int_{\Gamma_{1}} f_{\alpha} v_{\alpha} d s \text { for any } v \in V \text {, }
$$

where

$$
V=\left\{v \in H^{1}(\Omega), v=0 \text { on } \Gamma_{0}\right\} .
$$

It is classical that this problem is well posed for $\varepsilon>0$.

The equation and the boundary conditions equivalent to (5.5) are:

$$
\begin{aligned}
-\partial_{\beta} \sigma_{\alpha \beta}\left(u^{\varepsilon}\right) & =0 \text { in } \Omega, \\
u^{\varepsilon} & =0 \text { on } \Gamma_{0}, \\
\sigma_{\beta \alpha}\left(u^{\varepsilon}\right) n_{\beta} & =f_{\alpha} \text { on } \Gamma_{1},
\end{aligned}
$$

where $n$ denotes the outward unit normal.

Obviously, for $\varepsilon>0$, equation (5.7) is elliptic and both (5.8) and (5.9) satisfy the Shapiro-Lopatinsky condition. Moreover, in the limit case where $\varepsilon=0$ the system (5.7) is elliptic (in the Petrovskiu sense); the Dirichlet (fixation) condition satisfies the ShapiroLopatinsky condition, but the Neumann (free boundary) condition (5.9) does not. The problem is to study what happens as $\varepsilon \rightarrow 0$.

It is worthwhile writing explicitly $(5.7)-(5.9)$ in the limit case where $\varepsilon=0$. We have

$$
\begin{aligned}
-\left(\partial_{1}^{2}+\partial_{2}^{2}\right) u_{1} & =0, \quad-\left(\partial_{1}^{2}+\partial_{2}^{2}\right) u_{2}=0 \text { in } \Omega \\
u_{1}=u_{2} & =0 \text { on } \Gamma_{0}, \\
\partial_{1} u_{1} \cdot n_{1}+\left(\partial_{2} u_{1}+\partial_{1} u_{2}\right) n_{2} & =f_{1}, \quad\left(\partial_{2} u_{1}+\partial_{1} u_{2}\right) n_{1}+\partial_{2} u_{2} \cdot n_{2}=f_{2} \quad \text { on } \Gamma_{1} .
\end{aligned}
$$


We shall introduce the auxiliary unknowns $\sigma_{\beta \alpha}=\sigma_{\beta \alpha}(u)$. In fact, as in the case where $\sigma_{11}+\sigma_{22}=0$, as new auxiliary unknowns we shall take

$$
\sigma_{1}=\sigma_{11}(u)=-\sigma_{22}(u), \quad \sigma_{2}=\sigma_{12}(u) .
$$

Then problem (5.10)-(5.12) is precisely equivalent to

$$
-\partial_{1} \sigma_{1}-\partial_{2} \sigma_{2}=0, \quad \partial_{2} \sigma_{1}-\partial_{1} \sigma_{2}=0 \text { in } \Omega,
$$

with

$$
\sigma_{1} n_{1}+\sigma_{2} n_{2}=f_{1}, \quad-\sigma_{1} n_{2}+\sigma_{2} n_{1}=f_{2} \text { on } \Gamma_{1},
$$

or equivalently,

$$
\sigma_{1}=f_{1} n_{1}-f_{2} n_{2}, \quad \sigma_{2}=f_{1} n_{2}+f_{2} n_{1} \quad \text { on } \quad \Gamma_{1},
$$

and

$$
\partial_{1} u_{1}-\partial_{2} u_{2}=\sigma_{1}, \quad \partial_{2} u_{1}+\partial_{1} u_{2}=\sigma_{2} \text { in } \Omega,
$$

with

$$
u_{1}=u_{2}=0 \text { on } \Gamma_{0}
$$

Obviously, (5.13) is an elliptic system of first order with the unknowns $\sigma_{1}, \sigma_{2}$ (of total order 2), and (5.14) are Cauchy conditions for it. If we assume that $\sigma$ is known, then (5.15) becomes again an elliptic system of first order with the unknowns $u_{1}, u_{2}$ (of total order 2), and (5.16) are Cauchy conditions for it.

The standard properties of problem (5.7)-(5.9) may be summarized in the following way.

Proposition 5.1. System (5.7)-(5.9) possesses the following properties.

a) For $\varepsilon>0$ the problem is elliptic standard, with both variational and classical formulations. Existence and uniqueness hold true in standard spaces, without restrictions (i.e., the kernel is trivial and there are no compatibility conditions).

b) For $\varepsilon=0$ the system of equations is elliptic, but the boundary data (5.9) does not satisfy the Shapiro-Lopatinsky condition and the problem is out of the standard theory. Nevertheless, it enjoys uniqueness (in standard spaces).

c) If the boundary condition (5.9) is replaced by the Dirichlet one, i.e.,

$$
u=f \text { on } \Gamma_{1} \text {, }
$$

then for $\varepsilon>0$, part a) remains true. Moreover, for $\varepsilon=0$ the problem is elliptic standard (in the Agmon-Douglis-Nirenberg framework) and enjoys existence and uniqueness (the kernel is trivial and there are no compatibility conditions).

The conditions are obvious for $\varepsilon>0$ because of the variational formulation, which is coercive (classical). For $\varepsilon=0$ the variational formulation is valid, but coercivity is not proved. To prove uniqueness, we go back to the variational expression with $v=u$, which gives

so that $\widetilde{e}_{\alpha \beta}(u)=0$, i.e.,

$$
\int_{\Omega} \widetilde{e}_{\alpha \beta}(u) \widetilde{e}(u) d x=0
$$

$$
\partial_{1} u_{1}-\partial_{2} u_{2}=0, \quad \partial_{2} u_{1}+\partial_{1} u_{2}=0 \text { in } \Omega,
$$

which is a first order elliptic system. Then (5.8) plays the role of Cauchy conditions for it, and $u=0$ over $\Omega$. This follows from the standard local uniqueness theorem of Holmgren and analytic continuation. This result is independent of the boundary conditions on $\Gamma_{1}$, so that it holds true in both the b) and c) cases.

The only point that remains to be proved is existence without compatibility conditions in c). This follows from uniqueness, because the problem is selfadjoint. 
5.2. Reduction to a problem on the free boundary $\Gamma_{1}$. In order to exhibit the asymptotic behavior of the solution of problem (5.7)-(5.9), we can replace it by an equivalent problem, taking as unknowns the trace of $u^{\varepsilon}$ on $\Gamma_{1}$, denoted by $w^{\varepsilon}$. The idea is to solve the problem with the boundary condition (5.9) replaced by the Dirichlet condition $u^{\varepsilon}=w^{\varepsilon}$ on $\Gamma_{1}$, and then to write (5.9) with the right-hand side computed in terms of $w^{\varepsilon}$ by using the (nonlocal) resolvent. This leads to an equivalent formulation involving nonlocal operators on $\Gamma_{1}$. We proceed to do this.

For any $w^{\varepsilon} \in H^{s}\left(\Gamma_{1}\right)^{2}$, we solve the Dirichlet problem

$$
\begin{aligned}
-\partial_{\beta} \sigma_{\alpha \beta}\left(u^{\varepsilon}\right)=0 & \text { in } \Omega, \\
u^{\varepsilon}=0 & \text { on } \Gamma_{0}, \quad u^{\varepsilon}=w^{\varepsilon} \text { on } \Gamma_{1} .
\end{aligned}
$$

By part c) of Proposition 5.1 the solution is well defined for all $\varepsilon \geq 0$, the resolvent being continuous from $H^{s}\left(\Gamma_{1}\right)^{2}$ to $H^{s+1 / 2}(\Omega)^{2}$. Then, computing $\sigma_{\alpha \beta}\left(u^{\varepsilon}\right)$ from (5.3) and taking traces on $\Gamma_{1}$, we see that $(5.9)$ can be written as

$$
A^{\varepsilon} w^{\varepsilon}=f
$$

where $A^{\varepsilon}$ is continuous from $H^{s}\left(\Gamma_{1}\right)^{2}$ to $H^{s-1}\left(\Gamma_{1}\right)^{2}$. Obviously, $A^{\varepsilon}$ is not local, but the usual elliptic theory says that it is pseudolocal (the singular support of $A^{\varepsilon} w^{\varepsilon}$ is contained in the singular support of $w^{\varepsilon}$ ). Moreover, on an interval of values of $\varepsilon$ containing zero, $A^{\varepsilon}$ is a function of class $C^{\infty}$ with values in $L\left(H^{s}\left(\Gamma_{1}\right)^{2}, H^{s-1}\left(\Gamma_{1}\right)^{2}\right)$ (because the standard spectral theory for selfadjoint operators shows that the properties in Proposition 5.1 hold true) and may be prolongated for small negative $\varepsilon$.

Equivalence between (5.20) and the initial problem (5.7)-(5.9) via (5.18) is obvious, in accordance with existence and uniqueness in (5.18)-(5.19).

If $\varepsilon=0$ equation (5.20) has a somewhat nonclassical structure. Indeed, when $w^{\varepsilon}$ runs in $H^{s}\left(\Gamma_{1}\right)^{2}$, the corresponding $u$ exists in $H^{s+1 / 2}(\Omega)^{2}$ as well as $\sigma_{1}$ and $\sigma_{2}$, and their traces on $\Gamma_{1}$ are well-defined elements of $H^{s-1}\left(\Gamma_{1}\right)^{2}$. By construction, they are $A^{0} w$. Moreover, they constitute Cauchy data for the elliptic system (5.13), and accordingly, they should satisfy very drastic smoothness properties.

As a matter of fact, the equation $A^{\varepsilon} w^{\varepsilon}=f$ has the form

$$
\left(A^{0}+\varepsilon A^{1}\right) w^{\varepsilon}=f
$$

with operator $A^{1}$ elliptic of order 1 , whereas, according to the previous considerations, the limit problem has no solution for generic nonsmooth $f$.

Analyzing equation (5.21), we see that for $|\xi| \gg \log \varepsilon^{-1}$ this equation is elliptic of order one and the singularities of the solution $w^{\varepsilon}$ coincide with those of $f$. If $|\xi| \ll \log \varepsilon^{-1}$, the symbol of the operator $A^{\varepsilon}$ is exponentially decaying and the operator is smoothing. Thus, most important for the study is the transition zone, where $|\xi| \asymp \log \varepsilon^{-1}$, which corresponds to oscillation along the boundary $\Gamma_{1}$ with wave length proportional to $\left(\log \varepsilon^{-1}\right)^{-1}$.

\section{$\S 6$. Case of Dimension $n$ GReater than 2}

Here we consider a model problem in $\mathbb{R}^{n}$ with $n \geq 2$. Now the indices $\alpha, \beta, \ldots$ take values between 1 and $n$. Set

$$
e_{\alpha, \beta}=\frac{1}{2}\left(\partial_{\alpha} u_{\beta}+\partial_{\beta} u_{\alpha}\right), \quad \tilde{e}_{\alpha, \beta}=e_{\alpha, \beta}-\frac{1}{m} \delta_{\alpha, \beta} e_{\lambda, \lambda},
$$

where $m>0$ is a parameter. We have

$$
\partial_{\beta} \widetilde{e}_{\alpha, \beta}=\frac{1}{2} \Delta u_{\alpha}+\left(\frac{1}{2}-\frac{1}{m}\right) \partial_{\alpha} \operatorname{div} u .
$$


The system

$$
\partial_{\beta} \widetilde{e}_{\alpha, \beta}=f_{\alpha}
$$

is elliptic for $q=\frac{1}{2}-\frac{1}{m} \neq-1$, because its characteristic matrix is

$$
\left(\begin{array}{cccc}
|\xi|^{2}+q \xi_{1}^{2} & q \xi_{1} \xi_{2} & \ldots & q \xi_{1} \xi_{n} \\
q \xi_{1} \xi_{2} & |\xi|^{2}+q \xi_{2}^{2} & \ldots & q \xi_{2} \xi_{n} \\
\vdots & \vdots & \ddots & \vdots \\
q \xi_{1} \xi_{2} & q \xi_{1} \xi_{2} & \ldots & |\xi|^{2}+q \xi_{n}^{2} r
\end{array}\right)
$$

and the determinant is $|\xi|^{2 n}(1+q)$.

We show that the Shapiro-Lopatinsky conditions are satisfied. Let the normal to the boundary be $n=(1,0, \ldots, 0)$. Then on the free part of the boundary the conditions have the form

i.e.,

$$
\tilde{e}_{1, \beta}(u)=\psi_{\beta},
$$

If

$$
\partial_{1} u_{1}-\frac{1}{m} \partial_{j} u_{j}=\psi_{1}, \quad \frac{1}{2}\left(\partial_{1} u_{j}+\partial_{j} u_{1}\right)=\psi_{j}, \quad j=2, \ldots, n .
$$

$$
u=U e^{i \eta y+|\eta| x_{1}},
$$

where $y=\left(x_{2}, \ldots, x_{n}\right)$ and $\eta=\left(\xi_{2}, \ldots, \xi_{n}\right)$, then

$$
\left(1-\frac{1}{m}\right)|\eta| U_{1}-\frac{1}{m} i \eta_{j} U_{j}=\psi_{1}, \quad \frac{1}{2}\left(|\eta| U_{j}+i \eta_{j} U_{1}\right)=\psi_{j} .
$$

The matrix of this system is

$$
\left(\begin{array}{cccc}
\left(1-\frac{1}{m}\right)|\eta| & -\frac{1}{m} i \eta_{2} & \ldots & -\frac{1}{m} i \eta_{n} \\
\frac{i}{2} \eta_{2} & \frac{1}{2}|\eta| & \ldots & 0 \\
\vdots & \vdots & \ddots & \vdots \\
\frac{i}{2} \eta_{n} & 0 & \ldots & \frac{1}{2}|\eta|
\end{array}\right)
$$

and its determinant is $\left(1-\frac{2}{m}\right) 2^{1-n}|\eta|^{n}$.

On the other hand, if we replace system (6.1) with

$$
\partial_{\beta} \tilde{e}_{\alpha, \beta}+K \operatorname{div} u=f_{\beta},
$$

then the determinant of the system will be equal to $|\xi|^{2 n}(1+q+K)$, and the determinant of the system of boundary conditions becomes equal to

$$
\left(1-\frac{2}{m}+K\right) 2^{1-n}|\eta|^{n} \text {. }
$$

We arrive at the situation similar to that considered above if $m=2$ and $K=0$, or if $m$ is arbitrary but $K=\frac{2}{m}-1$.

\section{REFERENCES}

[1] Yu. V. Egorov and M. A. Shubin, Linear partial differential equations. Foundations of the classical theory, Partial Differential Equations - 1, Itogi Nauki i Tekhniki Sovrem. Probl. Mat. Fundam. Naprav., vol. 30, VINITI, Moscow, 1988, pp. 5-265; English transl., Foundations of the classical theory of partial differential equations, Encyclopaedia Math. Sci., vol. 30, Springer-Verlag, Berlin, 1998. MR1141629 (93a:35004c) MR1657445 (99f:35003)

[2] L. D. Landau and E. M. Lifshits, Theoretical physics. Vol. 7. Theory of elasticity, 4th ed., Nauka, Moscow, 1987; English transl., Course of theoretical physics. Vol. 7. Theory of elasticity, Pergamon Press, Oxford, 1986. MR0912888(88m:00005); MR0884707(88d:73001)

[3] F. Béchet, O. Millet, and E. Sanchez-Palencia, Singular perturbations generating complexification phenomena for elliptic shells, Comput. Mech. 43 (2009), 207-221. MR2453540 (2009j:74081) 
[4] Yu V. Egorov, N. Meunier, and E. Sanchez-Palencia, Rigorous and heuristic treatment of certain sensitive singular perturbations, J. Math. Pures Appl. (9) 88 (2007), 123-147. MR2348766 (2008m:35012)

[5] , Rigorous and heuristic treatment of sensitive singular perturbations arising in elliptic shells. Around the Research of V. Maz'ya, II, Springer-Verlag, Berlin, 2010, pp. 159-202. MR2676173

[6] R. F. Almgren, An anisotropic 3-dimensional structure with Poisson ration-1, J. Elasticity 15 (1985), 427-430.

[7] R. Lakes, Foam structures with negative Poisson ratio, Science AAAS 235 (1987), 1038-1040.

[8] V. M. Babich and V. S. Buldyrev, Asymptotic methods in short-wavelength diffraction problems. The method of canonical problems, Nauka, Moscow, 1972; English transl., Short-wavelength diffraction theory. Asymptotic methods, Springer Ser. on Wave Phenomena, vol. 4, Springer-Verlag, Berlin, 1991. MR0426630 (54:14569) MR.1245488 (94f:78004)

Laboratoire MiP, Université Paul Sabatier, 118 route de Narbonne, Toulouse 31062, FRANCE

E-mail address: egorov@cegetel.net

Laboratoire de Modélisation en Méchanique 4, Université Pierre et Marie Curie, place Jussieu, Case 162, Paris 75252, France

E-mail address: sanchez@lmm.jussieu.fr

Received 29/JUN/2010

Originally published in English 